Alaa A.-M. Abdel-Aziz*, Adel S. El-Azab, Hazem A. Ghabbour and Ahmad J. Obaidullaha

\title{
Crystal structure of $(E)-\mathrm{N}^{\prime}-((4-a m i n o p h e n y l)$ sulfonyl)-N,N-dimethylformimidamide, $\mathrm{C}_{9} \mathrm{H}_{13} \mathrm{~N}_{3} \mathrm{O}_{2} \mathrm{~S}$
}

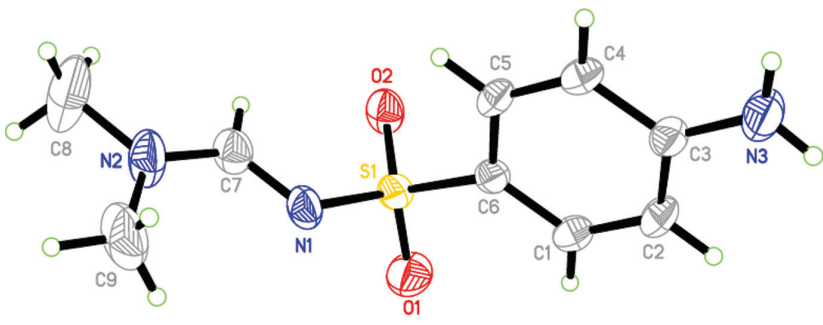

https://doi.org/10.1515/ncrs-2019-0781

Received October 20, 2019; accepted November 11, 2019; available online December 5, 2019

Table 1: Data collection and handling.

\begin{tabular}{ll}
\hline Crystal: & Colourless needle \\
Size: & $0.59 \times 0.13 \times 0.09 \mathrm{~mm}$ \\
Wavelength: & Mo $K \alpha$ radiation $(0.71073 \AA)$ \\
$\mu:$ & $0.27 \mathrm{~mm}^{-1}$ \\
Diffractometer, scan mode: & Bruker APEX-II, $\varphi$ and $\omega$ \\
$\theta_{\max }$, completeness: & $27.5^{\circ},>99 \%$ \\
$N(h k l)_{\text {measured }}, N(h k l)_{\text {unique }}, R_{\text {int }}:$ & $16215,2581,0.066$ \\
Criterion for $I_{\text {obs }}, N(h k l)_{\text {gt }}:$ & $I_{\text {obs }}>2 \sigma\left(I_{\text {obs }}\right), 1884$ \\
$N(\text { param })_{\text {refined }}:$ & 138 \\
Programs: & SHELX [1], Bruker [2]
\end{tabular}

\section{Abstract}

$\mathrm{C}_{9} \mathrm{H}_{13} \mathrm{~N}_{3} \mathrm{O}_{2} \mathrm{~S}$, monoclinic, $P 2_{1} / c$ (no. 14), $a=8.0984(4) \AA$, $b=17.3203(10) \AA, \quad c=9.6802(4) \AA, \quad \beta=124.031(3)^{\circ}$, $V=1125.26(10) \AA^{3}, Z=4, R_{\mathrm{gt}}(F)=0.0504, w R_{\text {ref }}\left(F^{2}\right)=0.1275$, $T=293(2) \mathrm{K}$.

\section{CCDC no.: 1514612}

The molecular structure is shown in the figure. Table 1 contains crystallographic data and Table 2 contains the list of the atoms including atomic coordinates and displacement parameters.

\section{Source of material}

A mixture of benzenesulfonamide ( $344 \mathrm{mg}, 2 \mathrm{mmol}$ ) and $\mathrm{N}, \mathrm{N}$-dimethylformamide dimethyl acetal (476 mg, $4 \mathrm{mmol}$ ) in ethanol $(10 \mathrm{~mL})$ was heated under reflux for $10 \mathrm{~h}$. The reaction mixture was cooled and the separated solid was filtered, dried and recrystallized from ethanol. Yield 96\%; ${ }^{1} \mathbf{H}$ NMR $\left(\right.$ DMSO- $\left.\mathrm{d}_{6} ; 500 \mathrm{MHz}\right): \delta 8.10(\mathrm{~s}, 1 \mathrm{H}, \mathrm{N}-\mathrm{C}(\mathrm{H})=\mathrm{N}), 7.39-7.37$

\footnotetext{
*Corresponding author: Alaa A.-M. Abdel-Aziz, Department of Pharmaceutical Chemistry, College of Pharmacy, King Saud University, P. O. Box 2457, Riyadh 11451, Saudi Arabia, e-mail: almoenes@ksu.edu.sa.https://orcid.org/0000-0002-33629337

Adel S. El-Azab and Ahmad J. Obaidullaha: Department of Pharmaceutical Chemistry, College of Pharmacy, King Saud University, P. O. Box 2457, Riyadh 11451, Saudi Arabia Hazem A. Ghabbour: Department of Medicinal Chemistry, Faculty of Pharmacy, University of Mansoura, Mansoura 35516, Egypt
}

Table 2: Fractional atomic coordinates and isotropic or equivalent isotropic displacement parameters $\left(\AA^{2}\right)$.

\begin{tabular}{lrrrr}
\hline Atom & $\boldsymbol{x}$ & $\boldsymbol{y}$ & $\boldsymbol{z}$ & $\boldsymbol{U}_{\text {iso }} / \boldsymbol{U}_{\text {eq }}$ \\
\hline S1 & $0.31663(8)$ & $-0.59517(3)$ & $-0.17899(7)$ & $0.03732(19)$ \\
O1 & $0.4058(3)$ & $-0.64624(11)$ & $-0.0384(2)$ & $0.0635(5)$ \\
O2 & $0.2455(2)$ & $-0.52250(9)$ & $-0.1591(2)$ & $0.0490(5)$ \\
N1 & $0.4747(3)$ & $-0.58306(11)$ & $-0.2299(3)$ & $0.0449(5)$ \\
N2 & $0.6689(4)$ & $-0.49539(14)$ & $-0.2558(3)$ & $0.0610(6)$ \\
N3 & $-0.3860(3)$ & $-0.74972(13)$ & $-0.7473(3)$ & $0.0613(7)$ \\
H2 & -0.4202 & -0.7928 & -0.7311 & $0.074^{*}$ \\
H3 & -0.4502 & -0.7290 & -0.8401 & $0.074^{*}$ \\
C1 & $0.0513(4)$ & $-0.71410(13)$ & $-0.3266(3)$ & $0.0438(6)$ \\
H1A & 0.1191 & -0.7371 & -0.2219 & $0.053^{*}$ \\
C2 & $-0.1152(4)$ & $-0.74905(13)$ & $-0.4584(3)$ & $0.0472(6)$ \\
H2A & -0.1592 & -0.7955 & -0.4417 & $0.057^{*}$ \\
C3 & $-0.2195(3)$ & $-0.71587(12)$ & $-0.6171(3)$ & $0.0388(5)$ \\
C4 & $-0.1487(3)$ & $-0.64599(12)$ & $-0.6387(3)$ & $0.0363(5)$ \\
H4A & -0.2143 & -0.6232 & -0.7436 & $0.044^{*}$ \\
C5 & $0.0161(3)$ & $-0.61130(12)$ & $-0.5066(3)$ & $0.0344(5)$ \\
H5A & 0.0602 & -0.5646 & -0.5224 & $0.041^{*}$ \\
C6 & $0.1189(3)$ & $-0.64450(12)$ & $-0.3492(3)$ & $0.0329(5)$ \\
C7 & $0.5336(4)$ & $-0.51297(15)$ & $-0.2254(3)$ & $0.0471(6)$ \\
H1 & 0.4779 & -0.4732 & -0.1997 & $0.057^{*}$ \\
C8 & $0.7321(7)$ & $-0.4158(2)$ & $-0.2474(7)$ & $0.1030(14)$ \\
H8A & 0.6694 & -0.3832 & -0.2094 & $0.154^{*}$ \\
H8B & 0.6943 & -0.3993 & -0.3561 & $0.154^{*}$ \\
H8C & 0.8742 & -0.4124 & -0.1713 & $0.154^{*}$ \\
C9 & $0.7652(5)$ & $-0.5543(2)$ & $-0.2925(5)$ & $0.0894(12)$ \\
H9A & 0.7915 & -0.5985 & -0.2234 & $0.134^{*}$ \\
H9B & 0.8883 & -0.5345 & -0.2708 & $0.134^{*}$ \\
H9C & 0.6797 & -0.5688 & -0.4076 & $0.134^{*}$ \\
\hline & & & &
\end{tabular}


(d, $2 \mathrm{H}, J=8.5 \mathrm{~Hz}, \mathrm{Ar}-\mathrm{H}), 6.58-6.56(\mathrm{~d}, 2 \mathrm{H}, J=9.0 \mathrm{~Hz}, \mathrm{Ar}-$ $\mathrm{H}$ ), $5.82\left(\mathrm{~s}, 2 \mathrm{H}, \mathrm{NH}_{2}\right.$ ), 3.10 (s, 3H, $\left.\mathrm{CH}_{3}\right), 2.87$ (s, 3H, $\left.\mathrm{CH}_{3}\right) \mathrm{ppm}$; ${ }^{13}$ C NMR (DMSO-d $\left.\mathrm{d}_{6} ; 125 \mathrm{MHz}\right): \delta 35.30\left(\mathrm{CH}_{3}\right), 41.14\left(\mathrm{CH}_{3}\right)$, 113.01, 128.20, 129.03, 152.41, $159.43(\mathrm{~N}-\mathrm{CH}=\mathrm{N})$ ppm; MS: $\mathrm{m} / \mathrm{z}=227.1$.

\section{Experimental details}

Cell refinement and data reduction were carried out by Bruker SAINT, SHELXT [1, 2].

\section{Comment}

Benzenesulfonamides $\left(\mathrm{ArSO}_{2} \mathrm{NH}_{2}\right)$ incorporating aromatic and heterocyclic ring systems are an important group of compounds, which have a wide range of biological activity such as anti-inflammatory, COX-1/2 inhibiting activity, inhibitors of the metalloenzyme carbonic anhydrase, and antitumor activities [3-8]. Recently, the synthesis of $N$-sulfonylformamidines has been reported through condensation of formamides and versatile sulfonamides using thionylchloride in the presence of chloroform as solvent [9]. The title compound was synthesized and reported according to method described by Silva, et al. [10]. Detailed synthetic studies indicate that this procedure gives the title compound.

The asymmetric unit of the title compound contains one independent molecule. The bond length between N1C7 is 1.296(3) $\AA$ which indicates that it is a double bond, compared to 1.320 (3) A between N2-C7. Bond lengths and angles of the title molecule are in the expected ranges [11]. The molecules pack in the crystal structure via two strong classical intermolecular hydrogen bonds, $\mathrm{N} 3-\mathrm{H} 2 \cdots \mathrm{N} 1^{\mathrm{i}}$ and $\mathrm{N} 3-\mathrm{H} 3 \cdots \mathrm{O} 1^{\mathrm{ii}}$ in addition to one non-classical hydrogen bond $\mathrm{C} 9-\mathrm{H} 9 \mathrm{~B} \cdots \mathrm{O} 2^{\mathrm{iii}}$. The $\mathrm{H} \cdot \cdots$ A distances are 2.31, 2.14 and $2.47 \AA$, respectively and the angles are 170,164 and $161^{\circ}$, respectively. Symmetry codes: (i) $x-1,-y-3 / 2, z-1 / 2$; (ii) $x-1, y, z-1$; (iii) $x+1, y, z$.

Acknowledgements: The authors acknowledge financial support from the Researchers Supporting Project number (RSP-2019/40), King Saud University, Riyadh Saudi Arabia.

\section{References}

1. Sheldrick, G. M.: SHELXT-integrated space-group and crystalstructure determination. Acta Crystallogr. A71 (2015) 3-8.

2. Bruker: APEX S, SADABS and SHELXT. Bruker-AXS, Madison, WI, USA (2014).

3. El-Azab, A. S.; Abdel-Aziz, A. A.-M.; Ayyad, R. R.; Ceruso, M.; Supuran, C. T.: Inhibition of carbonic anhydrase isoforms I, II, IV, VII and XII with carboxylates and sulfonamides incorporating phthalimide/phthalic anhydride scaffolds. Bioorg. Med. Chem. 24 (2016) 20-25.

4. Abdel-Aziz, A. A.-M.; El-Azab, A. S.; Ceruso, M.; Supuran, C. T.: Carbonic anhydrase inhibitory activity of sulfonamides and carboxylic acids incorporating cyclic imide scaffolds. Bioorg. Med. Chem. Lett. 24 (2014) 5185-5189.

5. Abdel-Aziz, A. A.-M.; El-Azab, A. S.; Ekinci, D.; Sentürk, M.; Supuran, C. T.: Investigation of arenesulfonyl-2imidazolidinones as potent carbonic anhydrase inhibitors. J. Enzyme Inhib. Med. Chem. 30 (2015) 81-84.

6. Alanazi, A. M.; El-Azab, A. S.; Al-Suwaidan, I. A.; ElTahir, K. E. H.; Asiri, Y. A.; Abdel-Aziz, N. I.; Abdel-Aziz, A. A.-M.: Structure-based design of phthalimide derivatives as potential cyclooxygenase-2 (COX-2) inhibitors: anti-inflammatory and analgesic activities. Eur. J. Med. Chem. 92 (2015) 115-123.

7. Abdel-Aziz, A. A.-M.; Angeli, A.; El-Azab, A. S.; Hammouda, M. E. A.; El-Sherbeny, M. A.; Supuranc, C. T.: Synthesis and anti-inflammatory activity of sulfonamides and carboxylates incorporating trimellitimides: dual cyclooxygenase/carbonic anhydrase inhibitory actions. Bioorg. Chem. 84 (2019) 260-268.

8. Abdel-Aziz, A. A.-M.; El-Azab, A. S.; El-Subbagh, H. I.; AlObaid, A. M.; Alanazi, A. M.; Al-Omar, M. A.: Design, synthesis, single-crystal and preliminary antitumor activity of novel arenesulfonylimidazolidin-2-ones. Bioorg. Med. Chem. Lett. 22 (2012) 2008-2014.

9. Hudabaierdi, R.; Wusiman, A.; Mulati, A.: Improved synthesis of $\mathrm{N}$-sulfonylformamidine derivatives promoted by thionyl chloride. Phosphorus, Sulfur Silicon Relat. Elem. 192 (2017) 485-489.

10. Silva, A. L.; Covarrubias-Zúñiga, A.; Maldonado, L. A.: A simple preparation of $N, N$-dimethyl- $N^{\prime}$-alkyl (aryl) sulfonylformamidines. Org. Prep. Proced. Int. 34 (2002) 545-549.

11. Zhang, X.; Cao, Y.; Wang, Y.; Wang, S.; Xu, Z.: The crystal structure of catena-poly[bis((4-aminophenyl)sulfonyl)(pyrimidin2-yl)amido- $\left.{ }^{2} N, N^{\prime}\right)$-bis $\left(\mu_{2}-4,4^{\prime}\right.$-bipyridine- $\left.{ }^{2} N: N^{\prime}\right) \operatorname{zinc}(I I)$ methanol (1/2), $\mathrm{C}_{32} \mathrm{H}_{34} \mathrm{~N}_{10} \mathrm{O}_{6} \mathrm{~S}_{2} \mathrm{Zn}$. Z. Kristallogr. NCS 233 (2018) 413-415. 